\title{
Photoconductive relaxation studies of SnSe thin films
}

\author{
T SUBBA RAO* and A K CHAUDHURI \\ Department of Physics, Indian Institute of Technology, Kharagpur 721 302, India \\ * Department of Physics, SV University PG Centre, Kurnool 518001, India
}

\begin{abstract}
The compound tin selenide was prepared from the constituent elements ( $\mathrm{Sn}$ and $\mathrm{Se}$ ) using the standard fusing technique and from $X$-ray diffraction studies it was identified as tin selenide. Thin films of SnSe were obtained on thoroughly cleaned glass substrates by vacuum sublimation on substrates maintained at $301 \mathrm{~K}$. The photoconductive relaxation of these films was studied with oxidation. The results have been explained with the help of grain boundary potential barrier model.
\end{abstract}

Keywords. Thin films; tin selenide; photoconductive relaxation; grain boundary potential barrier.

\section{Introduction}

Tin selenide whose room temperature indirect gap is about $0.9 \mathrm{eV}$, possesses some essential solar materials qualities (Loferski 1956, 1963; Rodot 1975, 1977). Photoelectric and optical studies of tin selenide polycrystalline films have been reported to some extent in literature (Bennouna et al 1983; Dang Tran Quan 1984; Subba Rao and Chaudhuri 1985; Engelken et al 1986). Charge carrier photorelaxation time is a basic parameter to characterize the photoelectronic properties of semiconductor. The present investigation was undertaken to study the photoconductance relaxation phenomenon in tin selenide polycrystalline thin films with oxidation as well as microstructural effects. The results have been explained with the help of grain boundary potential barrier. The photorelaxation time of charge carriers is found to lie between 50 and $150 \mathrm{sec}$ for as grown SnSe films deposited on glass substrates keeping the substrates at $301 \mathrm{~K}$ during deposition.

\section{Experimental}

To obtain thin films, the compound already prepared in a polycrystalline powder form was used. The polycrystalline powder was obtained after direct synthesis in an evacuated quartz ampoule $\left(10^{-4} \mathrm{~Pa}\right.$ ), the initial components ( $\mathrm{Sn}$ and Se) having a $5 \mathrm{~N}$ degree of purity ( $\mathrm{Sn}$ and Se) (Nuclear Fuel Complex, Hyderabad, India). The synthesis temperature was about $1133 \mathrm{~K}$. After $30 \mathrm{~h}$ of constant heating, it was quenched in water. The final compound was analysed with X-ray diffraction and showed that the structure was orthorhombic with lattice constants $a=11.4 \AA, b=4.16 \AA$ and $c=4.42 \AA$. On comparing with available data in literature (Agnihotri et al 1979), the compound was identified as tin selenide.

Evaporation was performed by ohmic heating. The deposition rate was about $30 \mathrm{~nm} / \mathrm{min}$, the thoroughly cleaned glass substrates being at $20 \mathrm{~cm}$ from the molybdenum boat. During deposition, pressure went up to $10^{-5}$ to $10^{-4} \mathrm{~Pa}$. Thickness of the samples were determined both by weighing and by interference methods.

Electrical conductivity and photoconductivity measurements were carried out in a conventional apparatus suitable for making measurements continuously from high 
temperatures to low temperatures ( 301 to $110 \mathrm{~K}$ ) (Subba Rao 1988). The amount of light falling on the sample during photoconductivity measurement was determined with the help of Eppley's calibrated thermopile. The source of light was a tungsten halogen lamp fed by a constant voltage supply. The light intensity of the order of $200 \mathrm{~mW} / \mathrm{cm}^{2}$ with tungsten halogen lamp $(600 \mathrm{~W}, 230 \mathrm{~V})$ in all cases was maintained at a constant level.

\section{Results and discussion}

The films deposited in vacuum and exposed to atmosphere after deposition are found to be $P$-type as determined by thermoelectric and Hall measurements. $X$-ray and electron diffraction studies revealed that these films consist of crystallites of different sizes ranging from $25 \mathrm{~nm}$ to $35 \mathrm{~nm}$ deposited on glass substrates at $301 \mathrm{~K}$ (Subba Rao

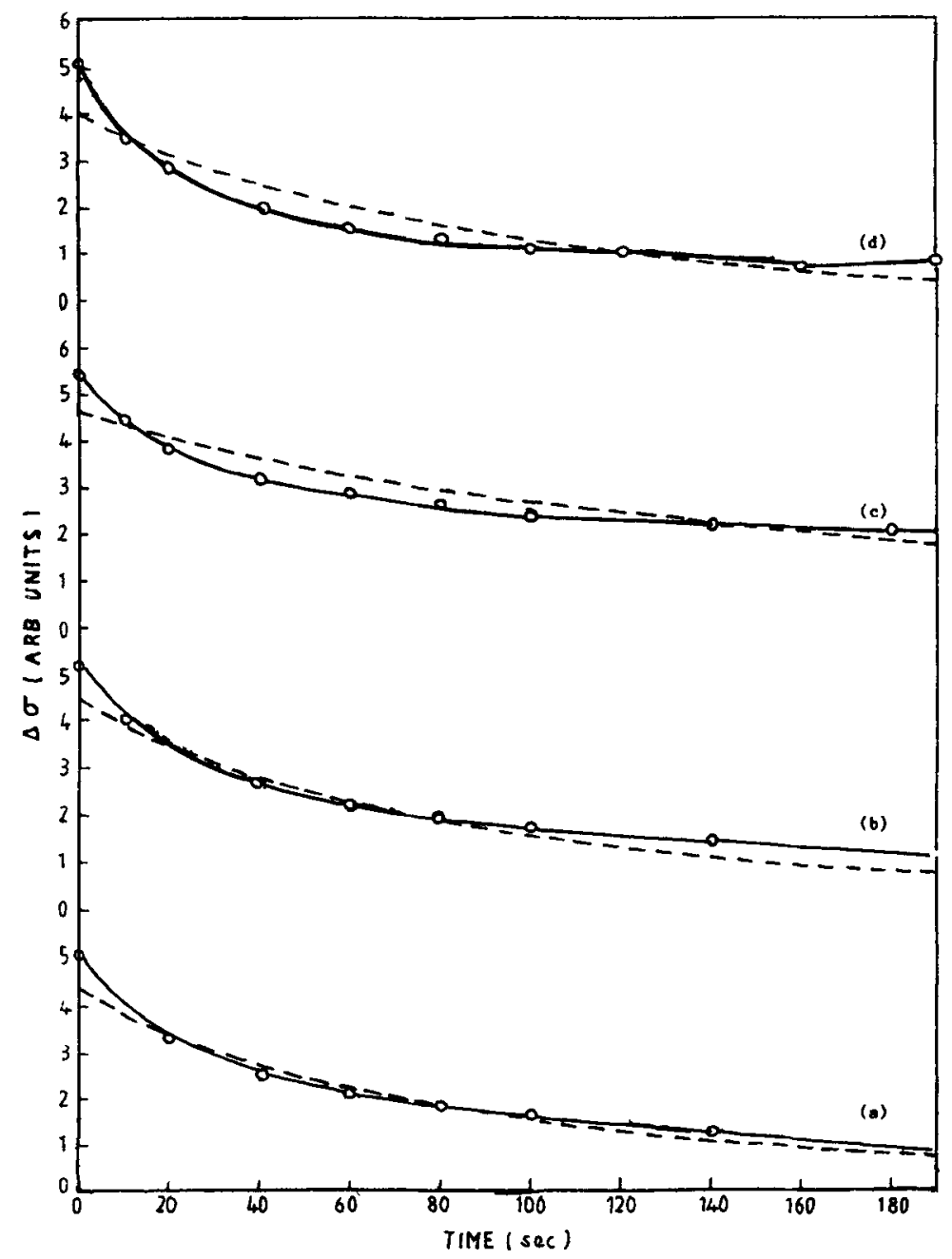

Figure 1. 


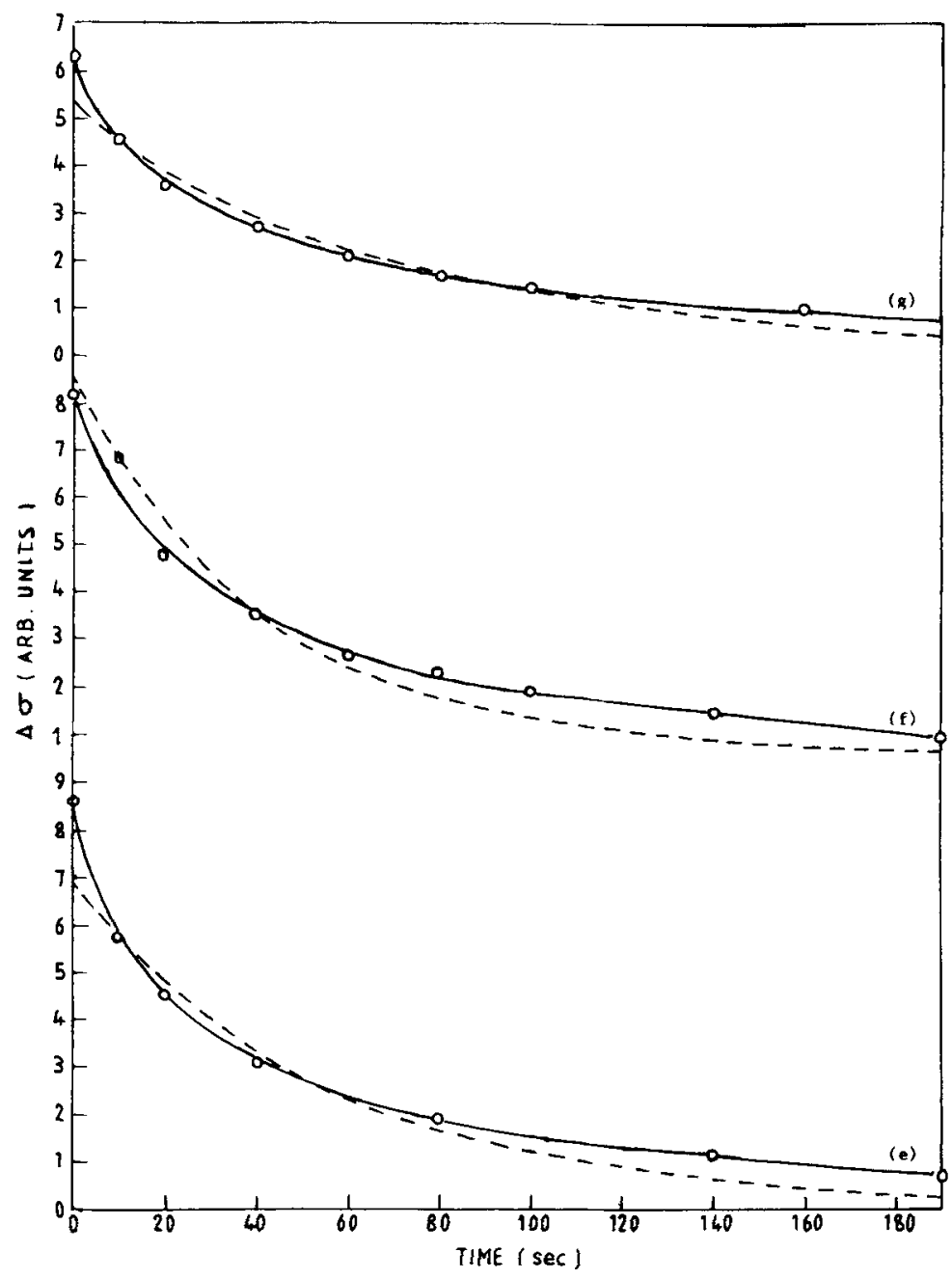

Figure 1. Photorelaxation curves of an as-grown film $(d=300 \mathrm{~nm})$ deposited on glass substrate and after aging for different periods of time. , Experimental results and -...-. theoretical results. (a) as-grown film, (b) after aging for $8 \mathrm{~h}$, (c) after aging for 2 days, (d) after aging for 24 days, (e) after aging for $\mathbf{4 0}$ days, (f) after aging for 31 days and (g) after aging for 36 days.

et al 1985). From the temperature variation of carrier concentration, the values of trap energy $\left(E_{\mathrm{t}}\right)$ are estimated. The values of $E_{\mathrm{t}}$ are found to lie between $0.062 \mathrm{eV}$ and $0.025 \mathrm{eV}$ (Subba Rao and Chaudhuri 1985). The value of grain boundary potential barrier estimated from the excess photoconduction as well as from the variation of mobility with temperature was found to be of the order of $0.17 \mathrm{eV}$ (Subba Rao and Chaudhuri 1985). Figure 1 shows the variation of photoconductance relaxation of $\mathrm{SnSe}$ as grown films with different aging times. From the experimental data it is apparent that the photorelaxation time constant increases with aging in these films (figure 2).

The effect of photoexcitation of tin selenide films can be described with the increase of carrier concentration in the grain in such films, when the light is put off the change of 


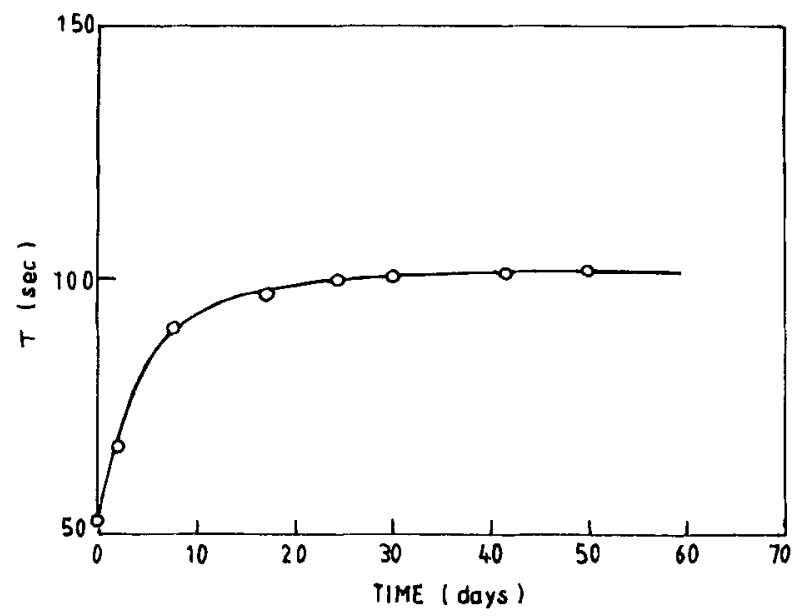

Figure 2. Variation of photoconductive relaxation time constant of as-grown film $(d=300 \mathrm{~nm})$ of $\mathrm{SnSe}$ with aging time.

Table 1. Aging time along with a photorelaxation time of charge carriers of SnSe thin films.

\begin{tabular}{lc}
\hline Aging time (days) & $\begin{array}{c}\text { Photorelaxation time }(\tau) \\
(\mathrm{sec})\end{array}$ \\
\hline As grown & 53 \\
2 & 67 \\
7 & 91 \\
24 & 100 \\
30 & 101 \\
90 & 143 \\
\hline
\end{tabular}

hole density in the valence band per unit time may be written as

$$
\frac{\mathrm{d} p}{\mathrm{~d} t}=-\gamma_{p}(p+\Delta p) \Delta p_{\mathrm{t}} e^{-\phi 1 / K T}
$$

where $\phi 1$ is the barrier height in $\mathrm{eV}$ under illumination and $\Delta p_{\mathrm{t}}$ the density of trapped carrier at the crystallite boundary.

For low level photoexcitation, (1) can be expressed as

$$
\frac{\mathrm{d} p}{\mathrm{~d} t}=-\gamma_{p}^{\prime} p \Delta p e^{-\phi 1 / K T},
$$

where $\gamma_{p}^{\prime}$ is the effective coefficient of capture of holes by traps. Solving this equation, we obtain

where

$$
\Delta p=\left(\Delta P_{0}\right) e^{-t / \tau}
$$

$$
\tau^{-1}=\gamma_{p}^{\prime} p e^{-\phi 1 / K T} \text {. }
$$


From expression (3), it is clear that the increase of $\tau$ with time is mainly due to the increase of grain boundary potential barrier with time due to diffusion of oxygen or oxidation at the grain boundary.

The variation of photorelaxation time $(\tau)$ with aging time is shown in table 1 . The effective coefficient of capture of holes by traps $\left(\gamma_{p}^{\prime}\right)$ for as grown SnSe film just after deposition is calculated from the relation (Seto 1975). The value of $\gamma_{p}^{\prime}$ obtained is observed to be of the order of $1.78 \times 10^{-21} \mathrm{~cm}^{3} / \mathrm{sec}$.

\section{Conclusions}

Tin selenide thin films were prepared by vacuum sublimation technique on glass substrates at $301 \mathrm{~K}$. The photoconductive relaxation studies reveal that the excess carrier generation was observed from the grain boundaries and the photorelaxation time increases with aging of the films.

\section{References}

Agnihotri D P, Jain A K and Gupta B K 1979 J. Cryst. Growth 46491

Bennouna A, Seignac A, Priol M, Dang Tran Q and Robin S 1983 Proc. of the 7th int. conf. on UUV-Anneals of the Israil Physical Society 6 p. 258

Dang Tran Q 1984 Phys. Status Solidi a86 421

Engelken R D, Berry A K, Van Doren T P, Boone J L and Shasnazary A 1986 J. Electrochem. Soc. 133581 Loferski J J 1956 J. Appl. Phys. 27777

Loferski J J 1963 Proc. IEEE 51667

Rodot M 1975 Acta Electronica 18345

Rodot M 1977 Rev. Phys. Appl. 12411

Seto J Y N 1975 J. Appl. Phys. 465247

Subba Rao T 1988 Studies on the structural, electrical and photo-electronic properties of tin selenide films, Indian Institute of Technology, Kharagpur

Subba Rao T and Chaudhuri A K 1985 J. Phys. D. Appl. Phys. 18 L35

Subba Rao T, Samantaray B K and Chaudhuri A K 1985 J. Mater. Sci. Lett. 4743 